\title{
Feature Extraction using Overlap Blocks for Content based Image Retrieval
}

\author{
Sinora Banker \\ Ghosalkar \\ TCET Kandivali (E) \\ Mumbai 400101
}

\author{
Vinayak A.Bharadi \\ TCET Kandivali (E) \\ Mumbai 400101
}

\author{
Sanjay Sharma \\ TCET Kandivali (E) \\ Mumbai 400101
}

Asif Ansari
Taif University
Saudi Arabia

\begin{abstract}
The paper presents an extension of content based image retrieval (CBIR) techniques based on Overlap Modified Block Truncation Coding (BTC) and Overlap Gabor Magnitude. Modified Block truncation coding based features is one of the CBIR methods proposed using color features of image. The approach basically considers red, green and blue planes of an image to compute feature vector. This MBTC based CBIR can be extended as Overlap Modified BTC for performance improvement in image retrieval. The Overlap Gabor Magnitude technique is used for texture features of image. The CBIR techniques like MBTC, Gabor and Fusion of MBTC and Gabor are further modified using overlap blocs technique to analyze and compare their performances. The proposed CBIR technique is implemented on a database having 1000 images spread across 11 categories and COIL image database having 1080 images spread across 15 categories. For each proposed CBIR technique 55 queries (5 per category) are fired on the database and net average precision and recall are computed. The results have shown performance improvement (higher precision and recall values) with Overlap (Fusion technique, Gabor Magnitude and Modified BTC compared to Non-Overlap (Fusion technique, Gabor Magnitude and Modified BTC ).In all Overlap technique based CBIR system gives best performance..
\end{abstract}

\section{General Terms}

Algorithm, Performance

\section{Keywords}

Content based image retrieval, Modified BTC, Gabor Magnitude, color, texture.

\section{INTRODUCTION}

A content-based image retrieval system (CBIRS) is a piece of software that implements CBIR. The creation of the World Wide Web has enabled millions of users to access data in a variant of media formats. This served as a stimulus for organizations having large image collections for e.g. fashion designers, newspaper editors etc to convert their collections to digital formats. This creates a need for development of techniques for efficient storage and retrieval of images.
In the past fifteen years ,many general- purpose image retrieval systems have been developed. Some of these are QBIC System, Photo book System, Blobworld System, Virage System ,Visual SEEK and Web SEEK Systems, the Pic Hunter System, NeTra System, MARS System, and SIMPLICITY Systems [1,2].

Existing CBIR systems can be grouped into two major categories: full-image retrieval system and region-based image retrieval system. Most of the existing CBIR systems are region-based systems because region-based systems are better than full-image retrieval systems.

In a CBIR System, to search images by their content, two things have to be done.

1. The image has to be re-encoded into some mathematical form and stored in a database.

2. There should be a mechanism to compare these mathematical forms.

Re-encoding is needed because an image is a collection of pixels with no meaning by itself. There is a gap between the visual information conveyed by the image and the way it is encoded. The process of re-encoding the image into a mathematical form suitable for comparison purpose is called feature extraction.

Features can also be grouped as low-level and high -level features. Low level features are features that can be obtained from the pixel itself. Examples are color and texture. High-level features are features obtained from the combination of low-level features. Examples are edge and shape. But, the three of the most widely used features for image retrieval are (i) color (ii) texture (iii) shape [3].

In the paper image retrieval method using Overlap Fusion CBIR technique is compared with Non-Overlap Fusion CBIR technique. Section 2 describes Current existing CBIR Techniques. Section 3 explains proposed CBIR techniques. Section 4 gives implementation and section 5 elucidates results with discussion. 


\section{CBIR IN EXISTING TECHNIQUES}

It is generally accepted that color and texture retrieval yield better results than shape matching [4].Image Retrieval using Spatial Block Truncation coding gives precision up to $100 \%$ than BTC and BTC LUV and Recall 100 [5],whereas image retrieval using Color Spatial gives precision upto78 \% to $89 \%$ and recall $100 \%$ [6]. This shows that BTC gives better results as it gives better compression ratio.

Part of the problem with shape matching lies in the difficulty of automatically distinguishing between foreground shapes and background detail in a natural image [1].Even when faced with stylized images or scenes where human intervention has been used to distinguish foreground from background, though, shape retrieval systems often perform poorly. A major contributing factor here is almost certainly the fact that few, if any, of the shape feature measures in current use are accurate predictors of human judgments of shape similarity [7]. Image retrieval using integrated color-shape location index fives precision $45 \%$ and Recall 100\% [8].

More recent techniques based on wavelets or Gaussian filtering appear to perform well in the retrieval experiments reported by their creators - though again, it is difficult to compare their effectiveness with more traditional methods, as no comparative evaluation studies have been performed. Content Based Image Retrieval Using Wavelet Transform gives Precision $71 \%$ for Recall $45 \%$ and Precision $19 \%$ for Recall $60 \%$ but it has ability to perform multiresolution decomposition. Modified BTC, Walsh Coefficients \& Histogram Moments Based Features for Content Based Image Retrieval gives moderate energy Compaction and Walsh Hadamard transform is very fast to calculate [9].

The volume of research into improved techniques for CBIR is increasing every year. Even allowing for the fact that much of it is concerned with minor modifications to existing methods, there is enough genuine innovation in areas such as semantic image retrieval, cross-media content description, and user interaction for us to be confident that there will be significant advances in commercially-available CBIR technology over the next ten years.

To obtain better result, we have proposed a better retrieval technique which integrated color and texture features in order to improve image retrieval. Modified Block Truncation Coding [10] is one of the common techniques used in image retrieval systems hence for our proposed system Modified Block Truncation Coding (MBTC) are used to combined with Euclidean distance in order to classify similar images for further enhancing the system ,texture features are extracted by using the concept of Overlap Gabor Magnitude. The result obtained by using this system is better than Non-Overlap Gabor Magnitude systems. Hence we can say that combination of color and texture feature with Overlap Gabor technique for finding similar image retrieval makes system more efficient and effective.

\section{METHOD}

\subsection{Modified Block Truncation Coding}

Block Truncation Coding, or BTC [11, 12] is a type of lossy image compression technique for grayscale images. It divides the original images into blocks and then uses a quantiser to reduce the number of grey levels in each block.
A $256 \times 256$ pixel image is divided into blocks of typically $4 \times 4$ pixels. For example, there is a $4 \times 4$ block from an image as shown in Figure 1.

\begin{tabular}{|l|l|l|l|}
\hline 245 & 239 & 249 & 239 \\
\hline 245 & 245 & 239 & 235 \\
\hline 245 & 245 & 245 & 245 \\
\hline 245 & 235 & 235 & 239 \\
\hline
\end{tabular}

Fig 1: Block of an image

For each block the Mean and Standard Deviation are calculated, these values change from block to block.

If a pixel value is greater than the mean it is assigned the value "1", otherwise " 0 ". Generate the binary map as shown in Figure 2 .

\begin{tabular}{|c|c|c|c|}
\hline $\mathbf{1}$ & $\mathbf{0}$ & $\mathbf{1}$ & $\mathbf{0}$ \\
\hline $\mathbf{1}$ & $\mathbf{1}$ & $\mathbf{0}$ & $\mathbf{0}$ \\
\hline $\mathbf{1}$ & $\mathbf{1}$ & $\mathbf{1}$ & $\mathbf{1}$ \\
\hline $\mathbf{1}$ & $\mathbf{0}$ & $\mathbf{0}$ & $\mathbf{0}$ \\
\hline
\end{tabular}

Fig 2: Binary Map

Divides whole image into $R, G$, and $B$ components.Computes interband average image (IBAI) which is the average of all components ( $R, G$, and $B$ )

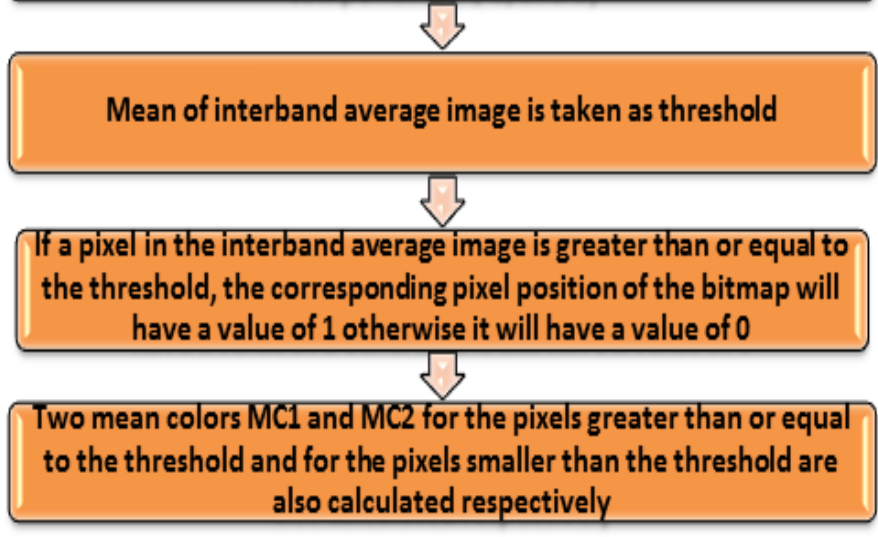

Fig 3: Basic Algorithm for Modified BTC

We consider image template of $256^{*} 256$ Pixels and find two mean colors for whole template and use them as a feature vectors.

The two mean colors are calculated as $\mathrm{MC} 1=\{\mathrm{Cr} 1, \mathrm{Cg} 1, \mathrm{Cb} 1\}$ and $\mathrm{MC} 2=\{\mathrm{Cr} 2, \mathrm{Cg} 2, \mathrm{Cb} 2\}[12]$.The two mean colors, Upper Mean and Lower Mean are computed. Now these Upper Mean and Lower Mean together will form a feature vector or signature 
of the image. The basic algorithm for Color feature extraction is shown in Figure 3.

\subsection{Gabor Magnitude}

Gabor filters are a group of wavelets, with each wavelet capturing energy at a specific frequency and a specific direction. Expanding a signal using this basis provides a localized frequency description, therefore capturing local features/energy of the signal. Texture features can then be extracted from this group of energy distributions. The scale (frequency) and orientation tunable property of Gabor filter makes it especially useful for texture analysis. The filters of a Gabor filter bank are designed to detect different frequencies and orientations. We use them to extract features on key points detected by interest operators [13].From each filtered image, Gabor features can be calculated and used to retrieve images. The procedure for extracting the Gabor feature vector is shown in following Figure 4 [12].

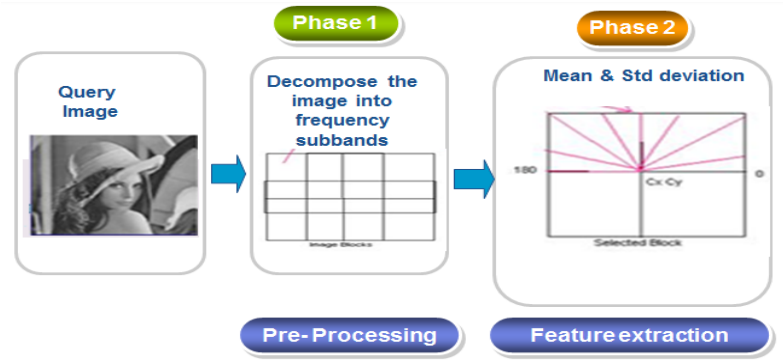

Fig 4: Gabor Filter Feature Extraction

For a given image $\mathrm{I}(\mathrm{x}, \mathrm{y})$, the discrete Gabor wavelet transform is given by a convolution:

$$
W_{m n}=\sum_{x_{1}} \sum_{y_{1}} I\left(x_{1}, y_{1}\right) g_{m n} *\left(x-x_{1}, y-y_{1}\right)
$$

Where $*$ indicates complex conjugate and where $\mathrm{m}, \mathrm{n}$ specify the scale and orientations of wavelet respectively.

After applying Gabor filters on the image with different orientation at different scale, we obtain an array of magnitudes:

$$
E(m, n)=\sum_{x} \sum_{y}\left|W_{m n}(x, y)\right|
$$

These magnitudes represent the energy content at different scale and orientation of the image. The main purpose of texture-based retrieval is to find images or regions with similar texture.

The standard deviation $\sigma$ of the magnitude of the transformed coefficients is:

$\sigma_{m n}=\sqrt{\frac{\sum_{x} \sum_{y}\left(\left|W_{m n}(x, y)\right|-\mu_{m n}\right)^{2}}{P \times Q}}$

Where $\mu_{m n}=\frac{E(m, n)}{P \times Q}$ is the mean of magnitude
A feature vector $\mathbf{f}$ (texture representation) is created using $m n$ and $m n$ as the feature components $[1,4] . M$ scales and $N$ orientations are used and the feature vector is given by:

$f=\left[\sigma_{00}, \sigma_{01} \ldots, \sigma_{(M-1)(N-1)}\right]$

$f_{\text {Gabor }}=\frac{f-\mu}{\sigma}$ where $\mu$ is the mean and $\sigma$ is the standard deviation of $f$.

\subsection{Overlap Technique}

Before utilizing the idea of Gabor Magnitude technique, the original image matrix is overlap blocked. The method is shown in Figure 5. The block size depends on the sampling point as shown. There is an overlap part between adjacent blocks. We could notice that every block of the image is related with each other. It improved the relationship among the adjacent pixels.

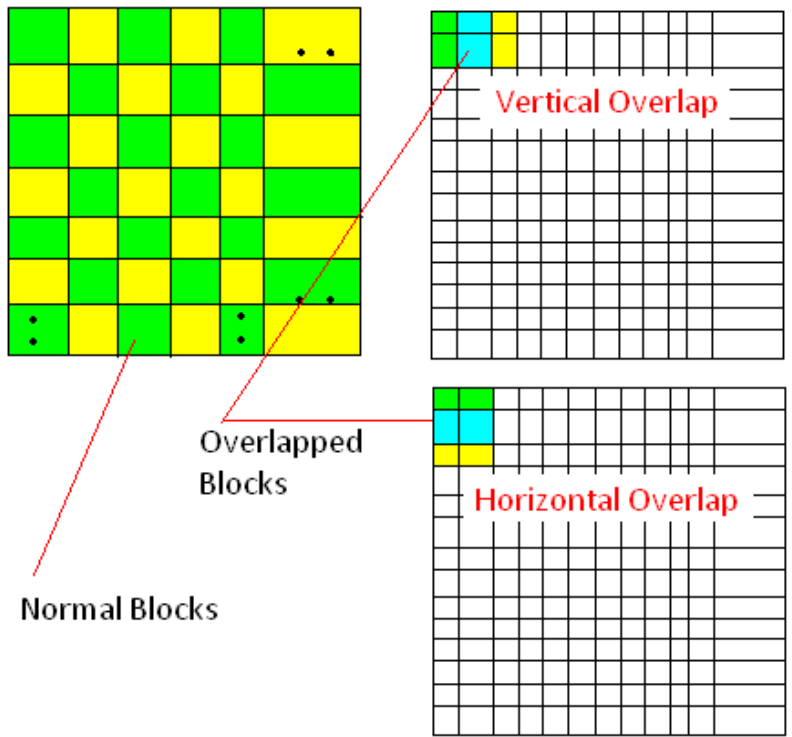

Fig 5: Representation of Overlap Block Method

Using Overlap Technique to extract the Texture feature is better way, here the window size increase as compared to previous implementation [12]. Here horizontal as well as vertical overlay is performed; blocks are overlapped by $50 \%$ in both vertical and horizontal direction. This method as marginal increase in the calculation but gives performance improvement. But in case of Non Overlap Gabor Feature extraction with block size of 16 x 16 we have 16 x 16 blocks. In case of overlapped with window size of 16 we have $16 \times 15$ such blocks. But the overlapped method gives us feature vector equivalent to $8 \mathrm{X} 8$ windows size, as the overlapping is $50 \%$. Thus we have finer details of the Gabor magnitude variations.

\section{IMPLEMENTATION}

In this approach feature vector of 6 components is formed by calculating Upper Mean and Lower Mean vector of R, G, and B planes of an image. Similarly by calculating Standard Deviation of Gabor Magnitude we obtain Gabor feature vector. The proposed CBIR technique is implemented in MATLAB on a database having 1000 images spread across 11 categories and 
COIL image database having 1080 images spread across 15 categories. For each proposed CBIR technique 55 queries ( 5 per category) are fired on the database and net average precision and recall are computed.

\subsection{Image Database}

The Generic database is downloaded from link mentioned here: http://wang.ist.psu.edu/ jwang/test1.tar. The COIL database http://www.cs.columbia.edu/CAVE/software/softlib/coil-100.php

.The categories and the distribution of the images are shown in Table 1 .

Table 1: Generic Database

\begin{tabular}{|l|c|}
\hline \multicolumn{2}{|c|}{ DATABASE IMAGE DISTRIBUTION } \\
\hline Number Of Images & Category \\
\hline Tribal People & 85 \\
Buses & 99 \\
Beaches & 99 \\
Dinosaurs & 99 \\
Elephants & 99 \\
Roses & 99 \\
Horses & 99 \\
Snow Mountains & 61 \\
Food & 100 \\
Historical Monuments & 99 \\
Sunrise Scenery & 61 \\
\hline
\end{tabular}

To compare the techniques and to check their performance we have used the precision and recall.

\subsection{Retrieval accuracy}

To assess the retrieval effectiveness, we have used the precision and recall as statistical comparison parameters for the MBTC and Gabor Filter [12].

It is given by

$$
\begin{gathered}
\text { Precision }=\quad \begin{array}{c}
\text { Number of relevant images retrieved } \\
\text { Total number of images retrieved }
\end{array} \\
\text { Recall }=\text { Total number of relevant images retrieved }
\end{gathered}
$$

\section{RESULTS AND DISCUSSION}

The methods Overlap-Gabor Magnitude and Fusion of Overlap Gabor Magnitude \& Modified BTC were applied to the image database having 1000 images spread across 11 categories and COIL image database having 1080 images spread across 15 categories. The average precision and average recall are computed by grouping the number of retrieved images sorted according to classification of database images with the query image.

\subsection{Generic Database}

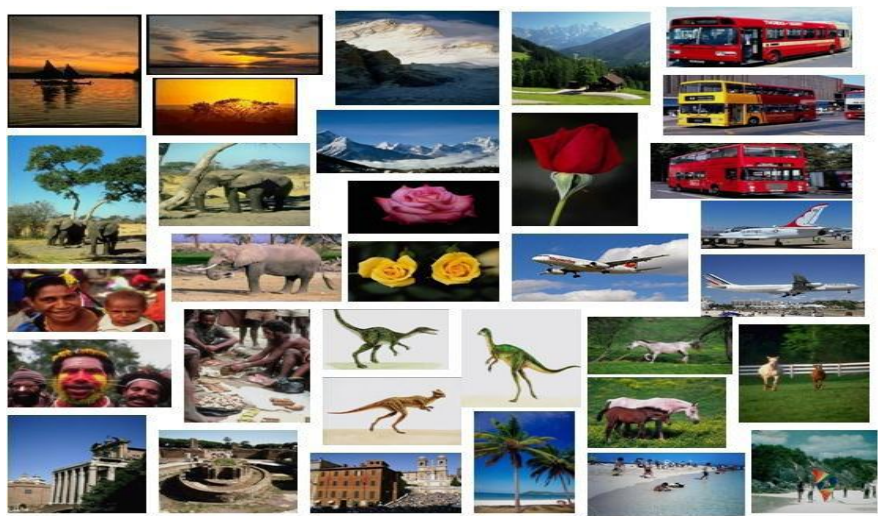

Fig 6 Sample images of Generic Image Database [Image database contains total 1000 images with 11 categories].

Figure 8 shows the graphs of precision/recall values plotted against number of retrieved images for all proposed image retrieval techniques. Here Overlap -Fusion based image retrieval technique gives the highest precision/recall crossover values specifying the best performance as compared with conventional Fusion based image retrieval technique [12]. The crossover point varies for different image category, and the highest crossover point 8.5 is achieved for Tribal People, Elephants, Roses and sunrise image category. Figure 9 shows the overlap technique implemented on generic database using Overlap Fusion technique which gives best results compared to previous results [12].It gives highest cross-over point of 0.85 .Figure 10 shows the overlap Gabor technique which gives better result than non-overlap Gabor technique. The cross-over point is 0.68 .

\subsection{COIL Database}

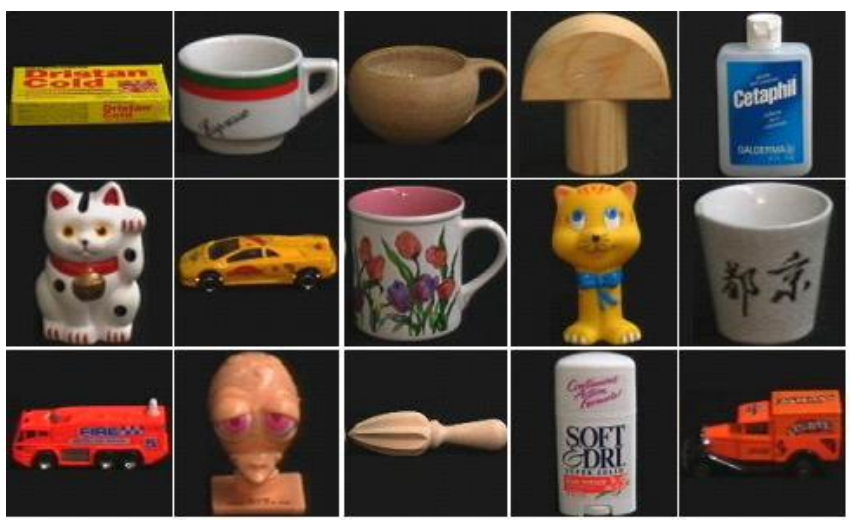

Fig 7. Sample of 15 images of COIL image database the database has 15 categories, for a total of 1,080 images 


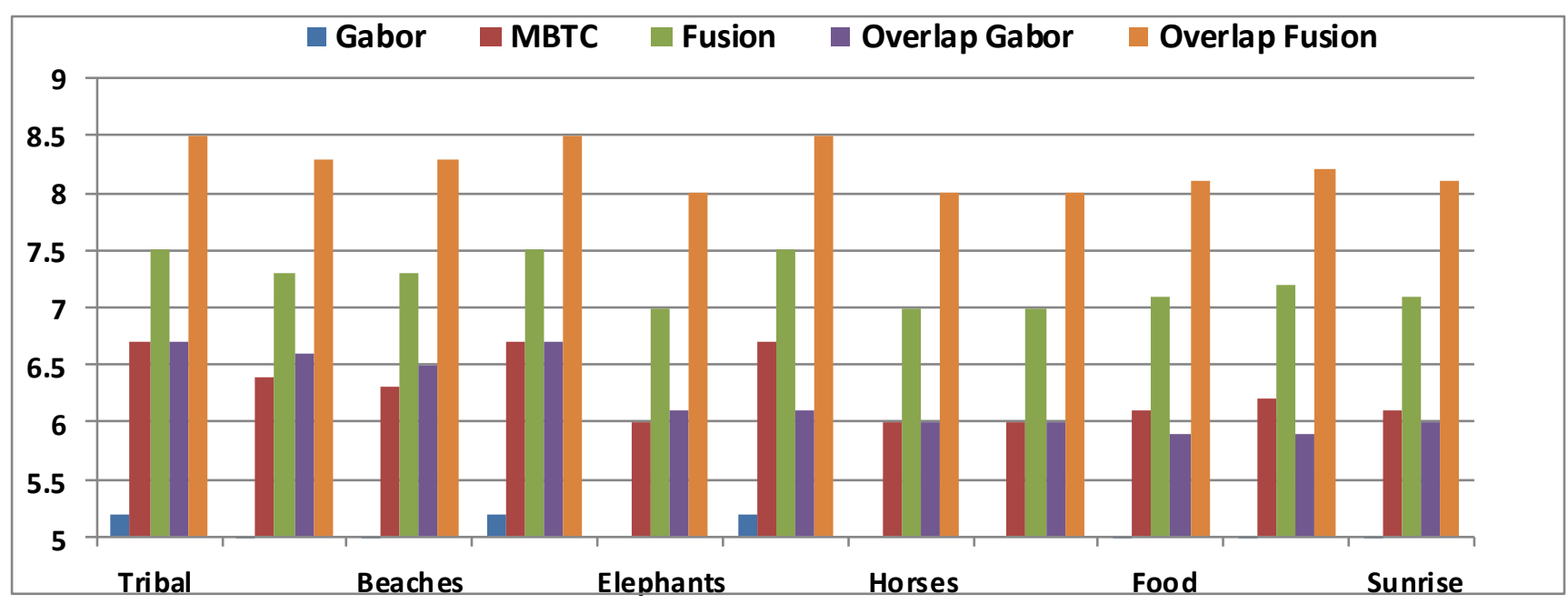

Fig 8 Crossover points for Precision \& Recall plotted against number of retrieve images for different image categories.(Generic

Database)

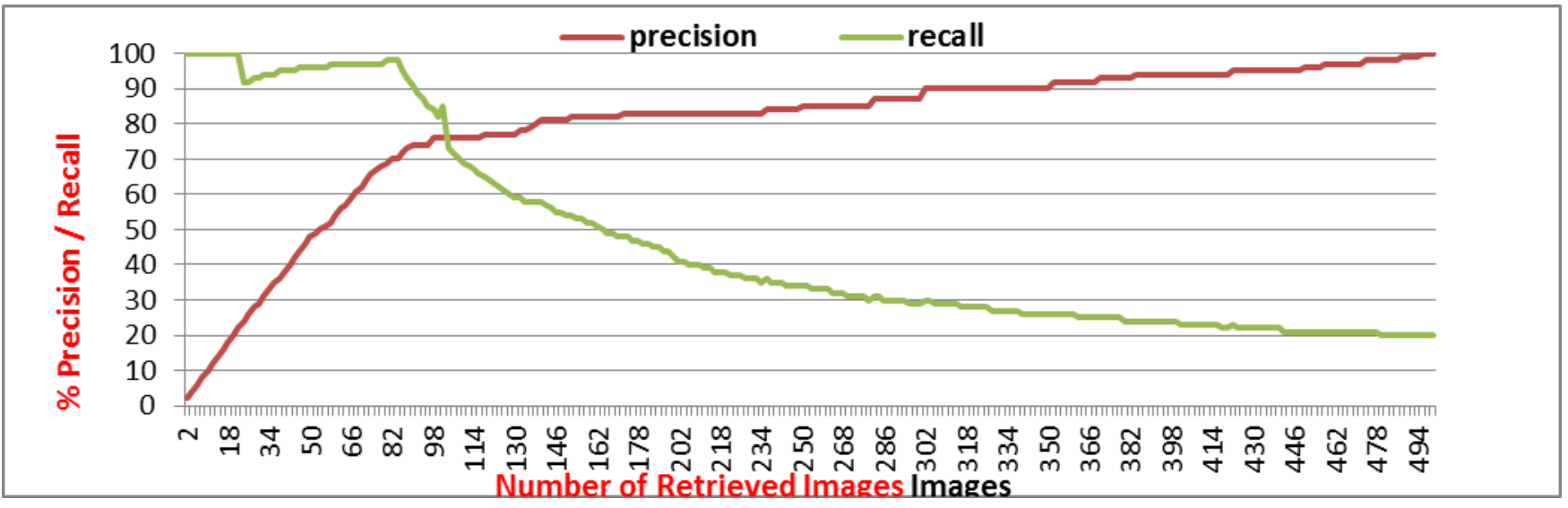

Fig 9 Precision \& Recall plotted against for Overlap Fusion based CBIR technique (Generic database)

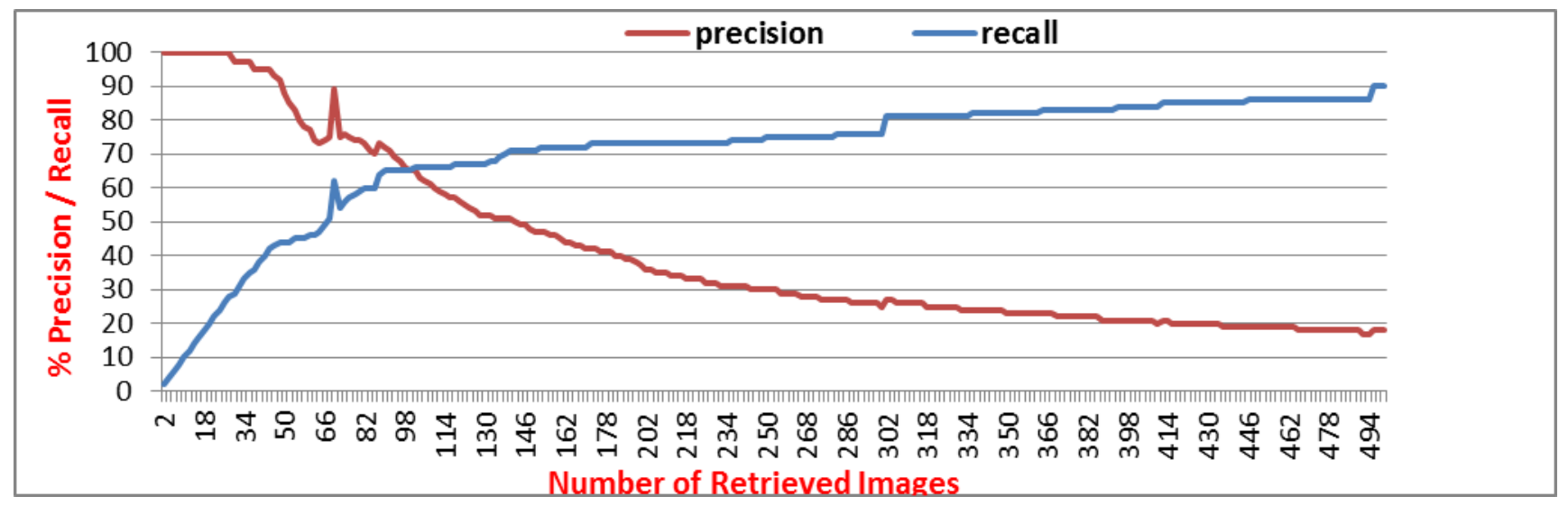

Fig 10. Precision \& Recall plotted against for Overlap Gabor based CBIR technique (Generic database) 


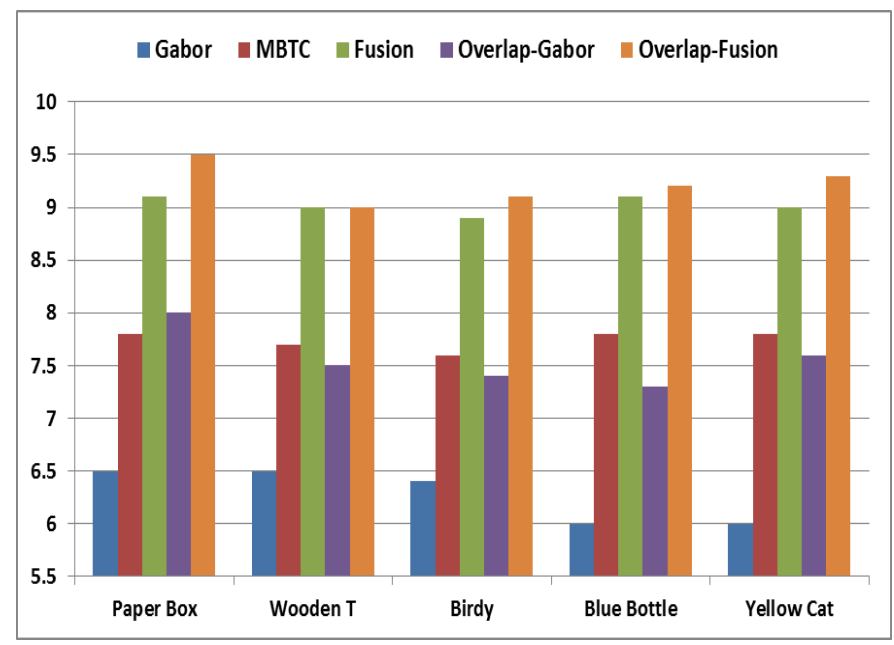

Fig 11 Crossover points for Precision \& Recall plotted against number of retrieve images for different image categories. (COIL Database).

Figure 11 shows the graphs of precision/recall values plotted against number of retrieved images for all proposed image retrieval techniques. Here Overlap -Fusion based image retrieval technique gives the highest precision/recall crossover values specifying the best performance. The crossover point varies for different image category, and the highest crossover point 9 is achieved for Paper Box, Wooden T, and Yellow Cat image category.

Figure 12 shows results obtained using Fusion technique tested on COIL image database. There is significant improvement in results using Fusion technique; the best results are obtained. Below precision-recall curve shows crossover point of 0.9 .

Figure 13 shows results obtained using MBTC based CBIR technique. There is no significant improvement in results for MBTC based CBIR technique alone. The crossover point for the MBTC based CBIR technique is 0.78.Figure 14 shows results obtained using Gabor based CBIR technique. There is no improvement in results using this technique alone; the results degrade slightly as no of the number of images go on increasing. The crossover point for the Gabor based CBIR technique is 0.68 .

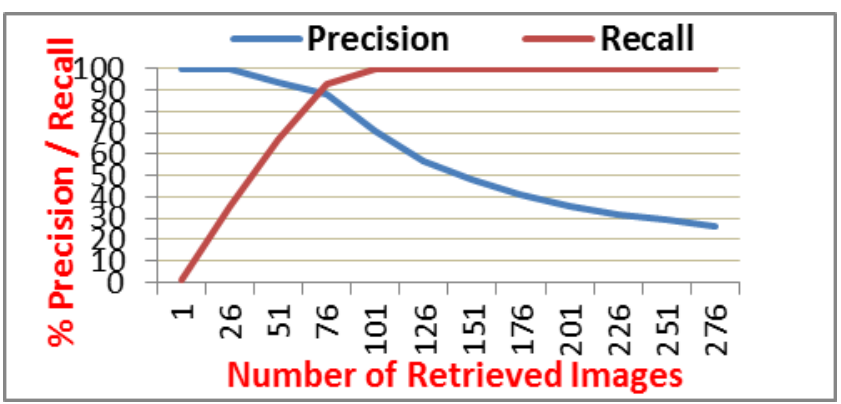

Fig 12 Precision \& Recall plotted against for Fusion based CBIR technique (COIL database).

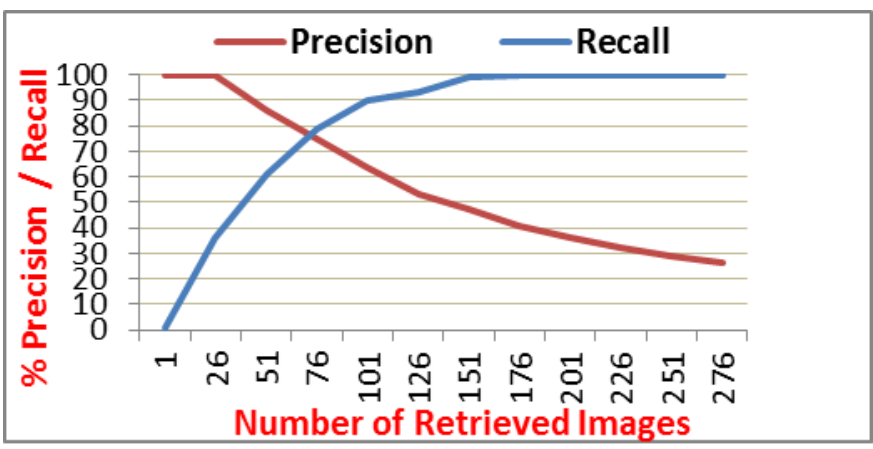

Fig 13 Precision \& Recall plotted against for MBTC based CBIR technique (COIL database).

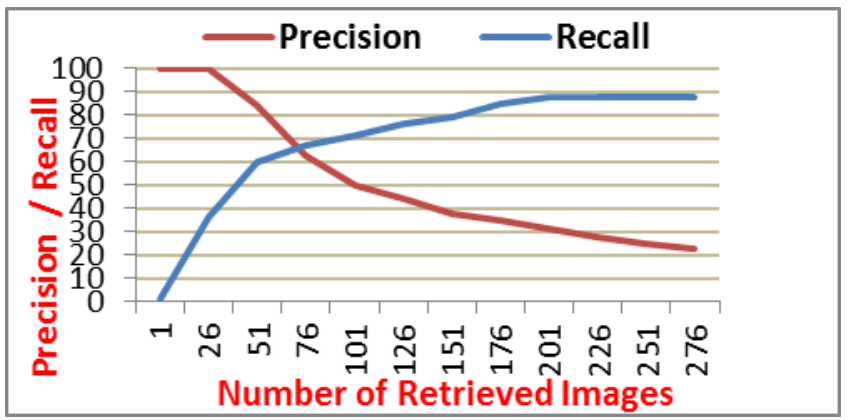

Fig 14 Precision \& Recall plotted against for Gabor based CBIR technique (COIL database).

Figure 15 shows the overlap technique implemented on coil database using Overlap Fusion technique which gives best results. It gives highest cross-over point of 0.95.Figure 16 shows the overlap Gabor technique which gives better result than nonoverlap Gabor technique. The cross-over point is 0.80 .

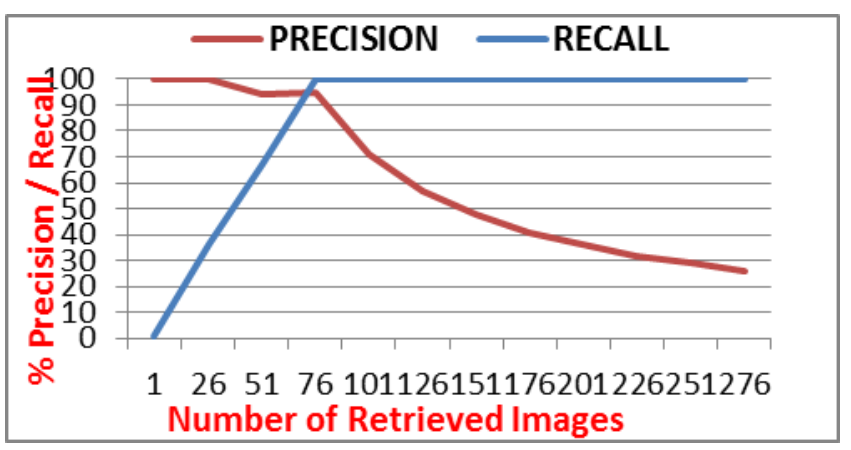

Fig 15 Precision \& Recall plotted against for Overlap Fusion Based CBIR technique (COIL database). 


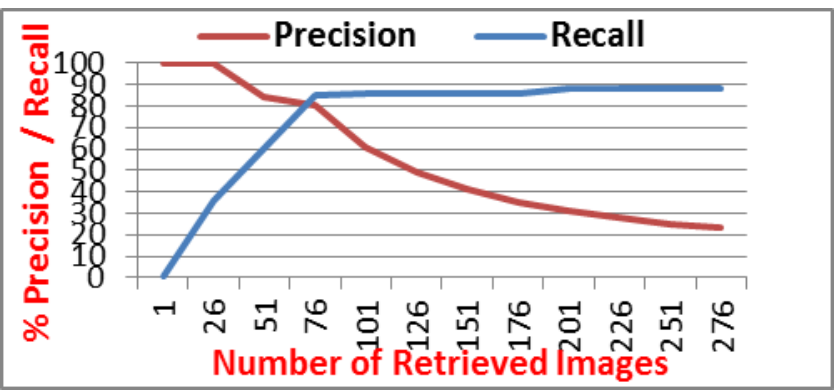

Fig 16 Precision \& Recall plotted against for Overlap Gabor Based CBIR technique (COIL database).

\section{CONCLUSION}

In this paper we have proposed a Content Based Image Retrieval System using Overlap technique based on Fusion of Gabor Filter's Response and Modified BTC of an image. The proposed system is giving higher Precision and Recall as compared to nonoverlap Fusion CBIR technique. The superiority of the system is because of the Gabor feature gives good response to texture of the image and Modified BTC give good response to color content of image.

\section{REFERENCES}

[1] Flickner M. et al, "Query by image and video content: the QBIC system” IEEE Computer 1995, 28(9), pp 23-32

[2] Gupta A. et al, "The Virage image search engine: an open framework for image management", in Storage and Retrieval for Image and Video.

[3] Dr. Fuhui Long, Dr. Hongjiang Zhang and Prof. David Dagan Feng,"Fundamentals of Content-Based Image Retrieval,"

[4] Faloutsos, C et al (1994) "Efficient and effective querying by image content" Journal of Intelligent Information Systems $3,231-262$.

[5] H.B.Kekre, Sudeep D. Thepade, "Boosting Block Truncation Coding with Kekre's LUV Color Space for Image Retrieval", International Journal of Electrical, Computer, and Systems Engineering 2;3 (c) www.waset.org Summer 2008.

[6] Guoping Qiu, "Color Image Indexing Using BTC,'IEEE Transactions on ImagProcessing, VOL.12, NO.1, pp.93-101, January 2003.

[7] Scassellati, B et al (1994) "Retrieving images by 2-D shape: a comparison of computation methods with human perceptual judgements" in Storage and Retrieval for Image and Video Databases II (Niblack, W R and Jain, R C, eds), Proc SPIE 2185, 2-14.

[8] B.G.Prasad, K.K. Biswas, and S. K.Gupta," Region -based image retrieval using integrated color, shape, and location index," computer vision and image understanding, October 2003.

[9] H B Kekre and V A Bharadi, "Modified BTC \& Walsh coefficients Based Features for Content Based Image Retrieval", Thadomal Shahani Engineering College, Bandra (East), Mumbai-51.

[10] Sinora Banker Ghosalkar,Vinayak A Bharadi,"Content-based Image Retrieval System",National ConferenceJabalpur,2009.

[11] Kai-Kuang Ma, Lei Huang, Shan Zhu, and Ho, A.T.S., "SPOT image compression using block truncation coding techniques " Geoscience and Remote Sensing, IGARSS '97, Remote Sensing - A Scientific Vision for Sustainable Development., 1997 IEEE International, Volume 4, pp.1996 - 1998, Aug. 1997.

[12] H.B. Kekre, V.A. Bharadi, S.D. Thepade, B.K. Mishra, S.E. Ghosalkar, S.M. Sawant, "Content Based Image Retreival Using Fusion of Gabor Magnitude and Modified Block Truncation Coding," icetet, pp.140-145, 2010 3rd International Conference on Emerging Trends in Engineering and Technology, 2010.

[13] ArtiKhaparde,,B.L.Deekshatulu,M.Madhavilath,ZakiraFarhe en,Sandhya Kumari V, "Content Based Image Retrieval Using Independent Component Analysis", IJCSNS International Journal ofComputer Science and Network Security, VOL.8 No.4, April 2000. 